\title{
Tratamiento de la dermatitis atópica. Una perspectiva desde la medicina basada en pruebas
}

\author{
JC. Buñuel Álvarez \\ Pediatra. ABS Girona-4 (Institut Català de la Salut). Girona
}

Rev Pediatr Aten Primaria. 2009; I I Supl I5:s69-s80

José Cristobal Buñuel Álvarez, jcbunuel@gmail.com

\section{Resumen}

En este artículo se resumen las principales intervenciones utilizadas para el tratamiento y prevención de la dermatitis atópica (DA). Para ello, se han revisado bases de datos secundarias y guías de práctica clínica de buena calidad metodológica con el objetivo de ofrecer una visión general de la eficacia de estas medidas. Para la redacción de este artículo se han utilizado principalmente dos recursos, Clinical Evidence y la guía de práctica clínica de NICE. Se ha complementado su información, cuando se ha estimado preciso, con otras fuentes de información, principalmente revisiones sistemáticas de la Colaboración Cochrane.

Palabras clave: Dermatitis atópica, Esteroides, Emolientes, Pimecrolimus, Lactancia materna, Probióticos.

\section{Abstract}

This article summarizes the most important interventions used in the treatment and prevention of atopic dermatitis (DA). Secondary databases and good methodological quality clinical guidelines have been reviewed in order to offer a general overview of the available evidence of these measures. Two resources have mainly been used: Clinical Evidence and the clinical guideline NICE.

Its information has been completed, when necessary, with other information resources, mainly systematic reviews of the Cochrane Collaboration. biotics.

Key words: Atopic dermatitis, Steroids, Emollients, Pimecrolimus, Breastfeeding, Pro-

\section{Introducción}

La dermatitis atópica (DA) es la enfermedad dermatológica más prevalente en la edad pediátrica. Se han publicado prevalencias que oscilan entre un $15-20 \%{ }^{1}$. Son muchos los tratamientos que son empleados para aliviar sus síntomas, así como para prevenir reagudizaciones de la enfermedad (RDA) (tabla I). 
La medicina basada en la evidencia o en pruebas se ha definido como "la utilización consciente, explícita y juiciosa de la mejor evidencia científica clínica disponible para tomar decisiones sobre el cuidado de cada paciente" $^{2,3}$. Puede ser considerada como una metodología de trabajo cuyo objetivo es conseguir el desarrollo de un criterio clínico personal en la toma de decisiones basadas en los resultados de la mejor investigación clínica realizada sobre pacientes (entendiendo como "mejor investigación" aquella que cumple unos criterios explícitos de validez metodológica y cuyos resultados pueden introducir cambios en la práctica clínica diaria).

El objetivo del presente artículo es efectuar una revisión de los diversos tratamientos utilizados en la DA, realizando para ello una búsqueda bibliográfica sistemática en aquellos recursos que tienen una mayor probabilidad de ofrecer información válida y relevante.

Una advertencia previa: " ausencia de evidencia" sobre un determinado tratamiento o medida preventiva no es sinónimo de "evidencia de ausencia" de efecto del mismo; se ha de tener presente que en el tratamiento de la DA, como en tantas otras enfermedades, no se han realizado ECC para comprobar la eficacia de todas las posibles intervenciones existentes. Es necesario, en estas ocasiones, complementar los resultados de los ensayos clínicos con los de otros tipos de estudios de tipo observacional e incluso, en ausencia de investigaciones sobre el tema, recurrir al consenso de expertos para establecer una recomendación útil para la práctica clínica diaria.

\section{Búsqueda bibliográfica}

Se efectuó una búsqueda en: 1) Clinical Evidence, revisándose el tema “Eczema (atopic)"4, publicado en 2005, cuya estructura servirá de guión para la redacción de este artículo; 2) TRIP Database, con los términos "atopic dermatitis" y "atopic eczema" restringidos al título de los documentos; 3) búsqueda en la Biblioteca Cochrane Plus, tanto en español (descriptores "dermatitis atópica" o "eccema atópico" restringidos al título del documento) como en inglés mediante la opción "Mesh" y el descriptor "atopic dermatitis"; 4) búsqueda en PubMed empleando los términos descriptores "atopic dermatitis" y restringiendo a referencias centradas en el tratamiento (búsqueda de metaanálisis $[M A]$, revisiones sistemáticas [RS] y, caso de no existir artículos con estos diseños, ECC). 
Resultados de la búsqueda

En Clinical Evidence existe un capítulo específico para DA4 . En TRIP Database se han recuperado 36 referencias (de ellas, 14 RS y 5 guías de práctica clínica [GPC]). En la Biblioteca Cochrane Plus se recuperaron 3, una de ellas ya dada de baja por no haber sido actualizada por sus autores. En PubMed se recuperaron 42 RS y MA. Restringiendo la búsqueda a los artículos publicados en los últimos 3 años, el número de referencias se reduce a 18 .

Después de una revisión de las diferentes referencias recuperadas, para la redacción de este artículo se ha tomado como referencia el capítulo "Eczema (atopic)" de Clinical Evidence, complementando su información con la proce- dente de GPC de calidad sobre el tema y con los resultados de RS recientes que realicen alguna aportación relevante.

\section{Intervenciones analizadas}

Como inicio de las intervenciones a evaluar, se partirá del cuadro resumen del capítulo de Clinical Evidence dedicado a DA adaptado a Pediatría, especificando las intervenciones que se valorarán (tabla I).

\section{Emolientes}

La revisión llevada a cabo por BathHextall et al. en 2005 concluyó que los emolientes son eficaces como tratamiento de primera línea aunque los estudios realizados para comprobar su efecto eran de pobre calidad metodo-

Tabla I. Dermatitis atópica: medidas terapéuticas y preventivas analizadas (Clinical Evidence, 2005)

\begin{tabular}{ll}
\hline Tipo de intervención & Caraterísticas de la intervención \\
\hline Medidas de autocuidado & Emolientes \\
\hline Medicamentos tópicos & Corticoides \\
& Pimecrolimus \\
& Tacrolimus \\
\hline Intervenciones dietéticas & Exclusión de alimentos \\
& Dieta elemental \\
& Probióticos \\
& Piridoxina \\
& Suplementos de zinc \\
& Ácidos grasos esenciales \\
\hline Medidas de prevención primaria & Lactancia materna prolongada \\
\hline Reducción de alergenos & Introducción precoz de probióticos \\
& Reducción de la exposición a alergenos (ácaros, restricción \\
& dietética materna) \\
\hline
\end{tabular}


lógica ${ }^{4}$. En general, cuanto más untuoso es el emoliente empleado mejores efectos se consiguen, aunque la consistencia del emoliente usado (demasiado graso) puede ser motivo de rechazo para su uso en adolescentes, sobre todo en zonas expuestas como cara y manos.

En los últimos 3 años se han publicado 2 ECC sobre la eficacia de diversos emolientes conteniendo glicerina al $20 \%{ }^{5}$ y avena ${ }^{6}$, constatándose un efecto beneficioso de los mismos frente a placebo. En el estudio de Grimalt et al. ${ }^{6}$ se informó además de una disminución del uso de corticoides tópicos en el grupo que recibió el emoliente.

Se recomienda que el uso de emolientes sea diario y frecuente (2 veces al día). En caso de ser aplicados por todo el cuerpo, pueden requerirse entre 250 $500 \mathrm{~g} / \mathrm{semana}$, según la edad y tamaño del niño ${ }^{4}$. El efecto adverso más frecuentemente relacionado con su uso es la sensación de ardor, siendo necesario en ocasiones cambiar de producto.

\section{Corticoides tópicos}

Bath-Hextall et al. ${ }^{4}$, después de revisar la bibliografía existente hasta 2005 con-

Tabla II. Clasificación de la gravedad de la dermatitis atópica y posible impacto sobre la calidad de vida (tomada de NICE*)

\begin{tabular}{|c|c|c|c|}
\hline \multicolumn{2}{|c|}{ Nivel de gravedad/alteraciones en la piel } & \multicolumn{2}{|c|}{$\begin{array}{l}\text { Impacto sobre la calidad de vida y el bienestar } \\
\text { psicosocial }\end{array}$} \\
\hline Normal & $\begin{array}{l}\text { Piel normal, ausencia de } \\
\text { pruebas de DA activa }\end{array}$ & Ninguno & $\begin{array}{l}\text { Ausencia de impacto } \\
\text { sobre la calidad de vida }\end{array}$ \\
\hline Leve & $\begin{array}{l}\text { Zonas de piel seca, picor } \\
\text { infrecuente (con o sin } \\
\text { pequeñas áreas } \\
\text { de enrojecimiento) }\end{array}$ & Leve & $\begin{array}{l}\text { Impacto pequeño sobre } \\
\text { las actividades cotidianas, } \\
\text { sueño y bienestar psicosocial }\end{array}$ \\
\hline Moderado & $\begin{array}{l}\text { Zonas de piel seca, picor } \\
\text { frecuente (con o sin excoriación } \\
\text { y engrosamiento localizado } \\
\text { de la piel) }\end{array}$ & Moderada & $\begin{array}{l}\text { Impacto moderado sobre las } \\
\text { actividades cotidianas, sueño } \\
\text { y bienestar psicosocial, } \\
\text { perturbación frecuente } \\
\text { del sueño }\end{array}$ \\
\hline Grave & $\begin{array}{l}\text { Amplias zonas de piel seca, } \\
\text { picor incesante, enrojecimiento } \\
\text { (con o sin excoriación, amplias } \\
\text { zonas de engrosamiento de la } \\
\text { piel, sangrado, piel agrietada y } \\
\text { alteración de la pigmentación) }\end{array}$ & Grave & $\begin{array}{l}\text { Limitación grave de las } \\
\text { actividades cotidianas } \\
\text { y de la función psicosocial, } \\
\text { pérdida de sueño nocturno }\end{array}$ \\
\hline
\end{tabular}


cluyeron que, comparados con placebo, los corticoides tópicos mejoran el aclaramiento de las lesiones en las RDA y disminuyen el porcentaje de recaídas de la enfermedad, aunque no hay acuerdo sobre el corticoide más eficaz ni sobre la posología más adecuada. Como recomendación, y dado el estado actual de conocimiento sobre el tema, se sugiere el uso inicial de corticoides de baja potencia una vez al día hasta 48 horas después de conseguir la remisión de las lesiones ${ }^{7}$ para proseguir, a continuación, tratamiento con emolientes ${ }^{4}$. En los estudios que se revisaron, la hidrocortisona a diferentes concentraciones (0,05-1\%) fue el corticoide de baja potencia más utilizado. El tratamiento corticoideo dependerá, en cualquier caso, de la gravedad de la DA (tabla II). En la tabla III se propone una aproximación terapéutica del uso de corticoides en función del nivel de gravedad de la DA basada en las recomendaciones de la GPC del NICE7. En la tabla IV se exponen los diferentes corticoides en función de su potencia. Se recomienda el uso de corticoides de baja potencia en cara y cuello. En caso de RDA graves, en estas zonas podrían utilizarse corticoides de potencia media durante no más de 3-5 días.

Una vez resueltas las lesiones agudas, en aquellos casos de RDA frecuentes pueden utilizarse corticoides de baja potencia, dos veces a la semana, para disminuir la incidencia de las reagudizaciones?.

Los efectos adversos de los corticoides tópicos pueden estar presentes en un $10 \%$ de los pacientes. Los más frecuentes son sensación de quemazón local, picor, foliculitis, hipertricosis, dermatitis de contacto y alteraciones de la pigmentación ${ }^{4}$.

\section{Inhibidores tópicos}

\section{de la calcineurina}

Los fármacos utilizados son el pimecrolimus al $1 \%$ y el tacrolimus al $0,03 \%$ y $0,1 \%$. No constituyen fármacos de primera elección en la DA, independientemente del nivel de gravedad de la misma. La FDA emitió un informe advirtiendo sobre un potencial riesgo canceríge-

Tabla III. Utilización de corticoides tópicos en función del nivel de gravedad de la dermatitis atópica

\begin{tabular}{lll}
\hline DA leve & DA moderada & DA grave \\
\hline Emolientes & Emolientes & Emolientes \\
\hline $\begin{array}{l}\text { Corticoides tópicos } \\
\text { de baja potencia }\end{array}$ & $\begin{array}{l}\text { Corticoides tópicos } \\
\text { de potencia media }\end{array}$ & $\begin{array}{l}\text { Corticoides tópicos } \\
\text { de alta potencia }\end{array}$ \\
\hline DA: dermatitis atópica. & & \\
\hline
\end{tabular}


no, basándose para ello en estudios realizados en animales, en la comunicación de algunos casos aparecidos en humanos y en el conocimiento del mecanismo de acción de estos medicamentos. Por ello, la FDA recomienda restringir su uso a aquellos casos en que los corticoides no han sido eficaces o bien no son aceptados. En cualquier caso, su uso no está admitido por debajo de los 2 años de edad. En la misma línea, la Agencia Española de Medicamentos y Productos
Sanitarios ha efectuado las siguientes recomendaciones 9 :

"El balance beneficio/riesgo de estos productos se mantiene favorable, aunque deben ser utilizados con gran precaución al objeto de reducir en lo posible el riesgo potencial de cáncer de piel y linfomas. Sobre la base de los datos actualmente disponibles, el Comité de Medicamentos de Uso Humano (CHMP) no puede concluir en qué medida el uso de pimecrolimus o tacrolimus puede aso-

Tabla IV. Potencia de los corticoides tópicos (fuente: NICE*)

\begin{tabular}{|c|c|}
\hline Potencia & Corticoide tópico \\
\hline Baja & $\begin{array}{l}\text { Desonida } 0,05 \% \\
\text { Acetato de hidrocortisona }(0,1-2,5 \%)\end{array}$ \\
\hline Media & $\begin{array}{l}\text { Dipropionato de alclometasona } 0,05 \% \\
\text { Valerato de betametasona } 0,025 \% \\
\text { Butirato de clobetasona } 0,05 \% \\
\text { Flurandrenolona } 0,0125 \% \\
\text { Acetonido de fluorcinolona } 0,00625 \% \\
\text { Butilesterato de flucortina } 0,75 \% \\
\text { Fluocortolona } \\
\text { Valerato de hidrocortisona } 0,2 \% \\
\text { Prednicarbato } 0,25 \%\end{array}$ \\
\hline Alta & $\begin{array}{l}\text { Dipropionato de beclometasona } 0,025 \% \\
\text { Dipropionato de betametasona } 0,05 \% \\
\text { Valerato de betametasona } 0,1 \% \\
\text { Valerato de flucortolona } 0,1 \% \\
\text { Acetonido de fluocinolona } 0,025 \% \\
\text { Fluocinonida } 0,05 \% \\
\text { Propionato de fluticasona } 0,05 \% \\
\text { Butirato de hidrocortisona } 0,1 \% \\
\text { Fuorato de mometasona } 0,1 \% \\
\text { Acetonido de triamcinolona } 0,1 \%\end{array}$ \\
\hline Muy alta & $\begin{array}{l}\text { Propionato de clobetasol } 0,05 \% \\
\text { Valerato de diflucortolona } 0,3 \% \\
\text { Halcinonida } 0,1 \%\end{array}$ \\
\hline
\end{tabular}


ciarse a los casos notificados de cáncer de piel y linfoma. El CHMP ha solicitado a las compañías titulares de la autorización de estos productos la obtención de mayor información relativa a la seguridad a largo plazo. El CHMP ha recomendado introducir cambios en la ficha técnica y el prospecto el fin de informar a médicos y pacientes sobre los potenciales riesgos a largo plazo asociados con el uso de estos productos. Las indicaciones autorizadas de pimecrolimus o tacrolimus después de la revisión realizada por el CHMP son las siguientes:

- Pimecrolimus: tratamiento en pacientes de 2 años de edad o mayores de la DA leve o moderada, cuando el tratamiento con corticosteroides tópicos no es aconsejable o no es posible. Esto puede ser debido a: intolerancia a corticosteroides tópicos, falta de efecto de corticosteroides tópicos, uso en la cara y cuello donde el tratamiento con corticosteroides tópicos puede ser inadecuado.

- Tacrolimus: tratamiento en adultos (pomada al 0,1 y al 0,03\%) o niños de 2 años de edad o mayores (únicamente pomada al 0,03\%) con DA moderada o grave que no responden adecuadamente o son intolerantes a los tratamientos convencionales como los corticoides tópicos".
Con fecha 22 de enero de 2009, el CHMP de la Agencia Europea del Medicamento ha emitido un informe positivo ${ }^{10}$ recomendando la modificación de los términos de uso comercial de la indicación de tacrolimus, en los siguientes términos: "Tacrolimus en pomada, dos veces/semana, previene los brotes y prolonga los periodos libres de síntomas entre brotes; este régimen está indicado para adultos y niños a partir de dos años, con dermatitis atópica moderada-grave que experimentan una elevada tasa de exacerbaciones de la enfermedad ( $\geq$ cuatro reagudizaciones/año), que previamente han presentado una buena respuesta a un tratamiento inicial con tacrolimus de una duración máxima de seis semanas (dos aplicaciones al día), entendiendo por buena respuesta un aclaramiento de las lesiones completo o casi completo, o afectación leve"10. En el mismo informe consta que "las condiciones detalladas para el uso de este producto serán publicadas en la actualización del "Summary of Product Characteristics", el cual será publicado en el "European Public Assessment Report" revisado y estará disponible en todos los idiomas oficiales de la Unión Europea, después de que la modificación para la autorización de comercialización haya sido concedida por la Comisión Europea"10. 
En la información dirigida a los pacientes, la Agencia Española de Medicamentos y Productos Sanitarios recomienda que estos fármacos pueden utilizarse bajo las siguientes condiciones:

1. Deben utilizarse según las indicaciones del médico y en el caso de que otras opciones terapéuticas no puedan aplicarse.

2. No deben utilizarse en niños menores de 2 años de edad.

3. La duración del tratamiento debe ser lo más corta posible para controlar los síntomas, aplicando una capa fina de producto exclusivamente en las zonas afectadas. A largo plazo el tratamiento debe ser intermitente.

4. Los pacientes actualmente en tratamiento no deben modificar ni suspender el mismo sin consultar con el médico.

En comparación con los corticoides tópicos, el pimecrolimus ha mostrado ser menos eficaz que los corticoides de potencia moderada o alta $^{11}$. Se desconoce su eficacia frente a corticoides de baja potencia debido a la falta de estudios que hayan comparado ambos productos. El tacrolimus al 0,1\% ha mostrado ser más eficaz que el pimecrolimus ${ }^{11}$.

Ambos productos, en comparación con placebo, presentan una mayor incidencia de efectos adversos leves locales (sensación de quemazón) ${ }^{4}$.

\section{Exclusión de alimentos}

Existe alguna evidencia, basada en estudios de pobre calidad metodológica, de que las fórmulas de hidrolizado de leche de vaca tienen una eficacia similar a las fórmulas basadas en aminoácidos para reducir la gravedad de la DA en niños con alergia probada a las proteínas de la leche de vaca4. Se han de tener en cuenta, al utilizar este tipo de productos, los riesgos que se asocian a una dieta exenta de productos lácteos: déficit de calcio y de aporte proteico y calórico ${ }^{4}$.

\section{Probióticos}

Bath-Hextall et al. ${ }^{4}$, en su revisión para Clinical Evidence concluyeron que hasta esa fecha (2005) no existían pruebas convincentes, basadas en ECC, que apoyaran el uso de probióticos para disminuir la sintomatología en niños con DA ya establecida. Asimismo, comprobaron que, en aquellos niños que recibieron lactobacilus inactivado se incrementó el riesgo de diarrea. Una revisión sistemática posterior ${ }^{12}$ ha constatado que no existen actualmente pruebas suficientes para recomendar el agregado de probióticos a la alimentación del recién nacido para la prevención de enfermedades alérgicas o de intolerancias alimentarias. Aunque existió una reducción en el eccema clínico en los neonatos, este efecto 
no fue consistente entre los estudios y se recomienda precaución en la interpretación de estos resultados debido a las debilidades metodológicas de los estudios incluidos.

\section{Otras intervenciones dietéticas}

En el tratamiento de la DA se han empleado otros alimentos o suplementos. En concreto, existen estudios sobre el uso de suplementos de zinc, de piridoxina y ácidos grasos esenciales. Ninguna de estas intervenciones ha mostrado ser eficaz, en comparación con placebo, para reducir la sintomatología de la $\mathrm{DA}^{4}$.

\section{Medidas de prevención primaria}

Bath-Hextall et al. ${ }^{4}$ concluyeron que la lactancia materna, en comparación con la leche de fórmula, puede reducir el riesgo de desarrollar DA. Esta conclusión se basó en estudios observacionales de tipo analítico debido a la ausencia de ECC que valoraran esta hipótesis.

\section{Reducción de alergenos}

No existen pruebas de que la restricción dietética materna durante el embarazo y lactancia de diversos alimentos potencialmente alergenos sea una medida eficaz para reducir la incidencia de DA en sus hijos durante los primeros 12-18 meses de vida. La restricción die- tética, por contra, podría producir una disminución de la ganancia de peso durante la gestación y asociarse a un mayor riesgo de parto pretérmino ${ }^{4}$.

Las medidas de reducción del polvo doméstico tampoco se han mostrado eficaces para prevenir el desarrollo de DA4.

\section{Introducción precoz de probióticos}

\section{en el último trimestre del embarazo}

Un ECC constató que la administración de probióticos 2-4 semanas antes del parto y hasta 6 meses después del mismo podía reducir la incidencia de DA entre los 2-4 años de edad, en comparación con placebo. Se ha de precisar que el ensayo se realizó en madres con riesgo elevado de tener un hijo atópico -en concreto, madres que tenían al menos un familiar de primer grado con DA-, por lo que habría que ser cautos a la hora de generalizar los resultados a la población general de madres sin predisposición para tener un hijo con enfermedad de tipo alérgico ${ }^{4,13}$.

\section{Conclusión}

El tratamiento de la DA se ha de basar en la aplicación de emolientes, independientemente del nivel de gravedad de la enfermedad. En función de la misma, pueden ir incorporándose fármacos tópicos, estando indicado el uso de corti- 
coides en las RDA. Aunque los estudios acerca de cuál es el corticoide más adecuado para el tratamiento son escasos y de baja calidad metodológica, parece razonable iniciar el tratamiento con corticoides de baja potencia y controlar la respuesta a los mismos. En el caso de que se consiga la remisión de las lesiones, el tratamiento corticoideo deberá suspenderse 48 horas después de la misma. En aquellos casos en que las RDA son frecuentes, se ha constatado que la aplicación semanal (2 veces/semana) de corticoides puede disminuir la incidencia de las reagudizaciones. Solo en aquellos casos en los que el paciente no obtenga mejoría alguna del tratamiento corticoideo tópico, y siempre que el niño sea mayor de 2 años, pueden estar indicados los inhibidores tópicos de la calcineurina que, en todo caso, solo se aplicarán en las zonas afectadas y se suspenderán en cuanto se obtenga la remisión de las lesiones. No existen en la actualidad pruebas que aconsejen otras medidas terapéuticas como suplementos de zinc, piridoxina o ácidos grasos esenciales. La administración de probióticos requiere de más investigaciones, bien diseñadas, para poder justificar su utilización en la prevención o el tratamiento de la enfermedad.

\section{Bibliografía}

1. Kay J, Gawkrodger DJ, Mortimer MJ, Jaron AG. The prevalence of childhood atopic eczema in a general population. J Am Acad Dermatol. 1994;30:35-9.

2. Sackett DL, Richardson WS, Rosenberg WMC, Haynes RB. Evidence-based Medicine. How to Practice \& Teach EBM. Nueva York: Churchill Livingstone; 1997.

3. Sackett DL, Rosenberg WMC, Muir Gray JA, Haynes RB, Richardson WS. Evidence based medicine: what it is and what it isn't. Br Med J. 1996:312:71-2.

4. Bath-Hextall F, Williams H. Eczema (atopic). Clinical Evidence. 2005 [en línea] [consultado el 10/06/2008]. Disponible en http://clinicalevi dence.bmj.com/ceweb/conditions/skd/1716/17 16-get.pdf

5. Breternitz $M$, Kowatzki $D$, Langenauer $M$, Elsner P, Fluhr JW. Placebo-controlled, doubleblind, randomized, prospective study of a glycerol-based emollient on eczematous skin in atopic dermatitis: biophysical and clinical evaluation. Skin Pharmacol Physiol. 2008;21:39-45.

6. Grimalt R, Mengeaud V, Cambazard F; Study Investigators' Group. The steroid-sparing effect of an emollient therapy in infants with atopic dermatitis: a randomized controlled study. Dermatology. 2007;214:61-7.

7. National Institute for Health and Clinical Excellence. Atopic eczema in children. Management of atopic eczema in children from birth up to the age of 12 years [en línea] [consultado el 12/06/2008]. Disponible en www.nice.org.uk/ guidance/index.jsp?action $=$ bylD\&o=11901 [actualizado en 12/2007]. 
8. FDA Public Health Advisory. Elidel (pimecrolimus) Cream and Protopic (tacrolimus) Ointment [en línea] [consultado el 10/06/2008]. Disponible en www.fda.gov/CDER/DRUG/advisory /elidel_protopic.htm

9. Agencia Española de Medicamentos y productos sanitarios. Nota informativa de 29 de marzo de 2006 [en línea] [consultado el 10/06/2008]. Disponible en www.agemed.es/ actividad/alertas/docs/NI_2006-3.pdf

10. European Medicines Agency. Evaluation of Medicines for Human Use. Nota informative del 22 de enero de 2009 [en línea] [consultado el 25/4/2009]. Disponible en www.emea.europa.eu /pdfs/human/opinion/Protopic_4034009en.pdf.

11. Ashcroft DM, Chen LC, Garside R, Stein K, Williams HC. Pimecrolimus tópico para el eccema (Revisión Cochrane traducida). En: La Biblio- teca Cochrane Plus, 2008 Número 1. Oxford: Update Software Ltd. Disponible en: http:// www.update-software.com. (Traducida de The Cochrane Library, 2008 Issue 1. Chichester, UK: John Wiley \& Sons, Ltd.).

12. Osborn DA, Sinn JK. Probióticos para la prevención de la enfermedad alérgica y la intolerancia alimentaria en neonatos (Revisión Cochrane traducida). En: La Biblioteca Cochrane Plus, 2008 Número 1. Oxford: Update Software Ltd. Disponible en: http://www.update-software. com. (Traducida de The Cochrane Library, 2008 Issue 1. Chichester, UK: John Wiley \& Sons, Ltd.).

13. Kalliomaki M, Salminen S, Pousa T, Arvilom$\mathrm{mi} \mathrm{H}$, Isolauri E. Probiotics and prevention of atopic disease: 4-year follow-up of a randomised placebo-controlled trial. Lancet. 2003;361:1869-71. 